\title{
ENTREVISTA A FRANCISCO HUENCHUMILLA
}

Fernando Pairican Padilla 


\title{
FRANCISCO HUENCHUMILLA, PRIMER SENADOR MAPUCHE: "LA AUTONOMÍA ES ALGO QUE ME ATRAE, CREO QUE PUEDE SER UN GRAN AVANCE PARA CHILE”
}

\author{
Por Fernando Pairican \\ Candidato a Doctor en Historia por la Universidad de Santiago de Chile. \\ Miembro de la Comunidad de Historia Mapuche y director de la colección \\ de Pensamiento Mapuche Contemporáneo de Pehuen Editores
}

Francisco Huenchumilla se ve contento. Asume que es el primer senador en la bistoria del pueblo mapuche, a pesar de que no le gusta que lo encasillen en una sola identidad. Su victoria no fue simple y la principal competencia vino desde su mismo partido con la figura de Fuad Chabin, quien a eso de las 21 horas del 19 de noviembre lo sobrepasó por un estrecho margen. El Partido Demócrata Cristiano, PDC, en el que Huenchumilla ha militando toda su vida, se inclinó por Chabin en las elecciones senatoriales, dejando entrever, entre otras aristas, las pugnas que al interior del histórico partido se han visualizado durante los últimos meses. Sin embargo, como ha quedado demostrado en su paso por el Gobierno, Huenchumilla sabe de desafíos y de remar contra la corriente, y comeñó a superar a Chabin a eso de la medianoche. "No dormi esa noche, destapé la champagne a eso de las cinco de la madrugada", una vez que la tendencia le brindó el triunfo con un 11,30\%, dice. Según él, fueron las zonas urbanas, Temuco en especial, comuna de la que fue alcalde, las que le dieron la victoria. Sin embargo, también plantea que las comunas con alto porcentaje de población mapuche le brindaron su apoyo. El senador avizora que tras su periodo de ocho años se retirará de la política y que espera dejar un "sello" como el primer senador de las "primeras naciones", una cuestión significativa en el escenario actual, marcado por los cuestionamientos a la "Operación Huracán", a la que se refiere como la culminación del error de "entregarle a Carabineros y al Ministerio Público la solución de un tema político".

\section{¿Se considera el primer senador mapuche de la historia?}

Efectivamente, soy el primer senador mapuche de la historia. En Chile, pertenecer al mundo de las primeras naciones se hace cuesta arriba en todos los ámbitos, y en el mundo de la política también, siempre se juega como de "visita". La política es un mundo, un lugar muy difícil para todos, sobre todo si esa persona se llama Huenchumilla. Se debe luchar contra todo: contra los poderes fácticos que no quieren que tú seas senador por las características que uno tiene. No es fácil que las personas de las zonas urbanas quieran votar por los mapuche. Uno no sale sólo por el voto mapuche, uno debe tener un ancla. Ese fue para mí un largo trabajo, es la coronación de un largo trabajo como abogado, diputado, alcalde. He pasado por 
muchas pruebas de blancura para tener una imagen, tener otra opinión pública que en definitiva me dio el triunfo. Fue una tarea gigantesca y hemos logrado clavar en la historia de Chile un hito que marca qué oportunidades van a tener en el Senado las personas de origen mapuche.

Usted ha planteado que su identidad también es Jaramillo, chilena, y que no le gusta que lo encasillen como mapuche. Sin embargo, es difícil no responder a eso desde una postura étnica. ¿Cuál es su posición frente al movimiento autodeterminista? ¿Piensa en algún proyecto de ley para colaborar en la lucha por los derechos colectivos de nuestro movimiento mapuche?

Mi mirada siempre es política. Como senador provengo de un pueblo que fue derrotado por el Estado y despojado de sus tierras por el Estado. Un pueblo que se para frente a él para reivindicar aquel acto. Entonces, el retroceso del pueblo mapuche en función de lo que tenía hace 140 años es el meollo de la reivindicación frente al Estado. Esa situación, ese escenario, requiere de un pueblo que se ponga de pie y que sea activo. Ese es un acto indispensable y por ende, ningún diputado o senador hará nada si un pueblo no exige un cambio de estatus con relación al Estado y la sociedad.

Ahora, en el actual sistema, pensar que un senador puede cambiar algo es muy difícil. El sistema político limita cualquier movimiento, ya que el Estado es quien conduce. No obstante, uno puede presentar proyectos de ley para hacer hechos políticos; yo los presenté como diputado para escaños reservados y, por supuesto, ahora presentaré un proyecto para que quede en la historia y en las negociaciones a futuro sirva como insumo para lograr un Estado Plurinacional y el reconocimiento de los derechos colectivos del pueblo mapuche. Será un proyecto acorde con las leyes internacionales. En esa línea lo voy a presentar; seguramente no me lo van a recibir con los brazos abiertos, pero creo que es una obligación hacerlo para contribuir al debate, porque es una lucha larga. Y lo otro, voy a estudiar los efectos que se hacen con la restitución de tierras y las formas que tiene el Estado para afrontar ese escenario diferente. Cómo se administra, cómo se gestiona, cómo es la organización frente al mundo de la economía. Todo eso está en deuda. Cómo se gestiona, cómo lo vamos a repartir. Hay todo un mundo jurídico, político respecto a ese punto. No se han hecho estudios sobre eso.

\section{Un proyecto de ley sobre autodeterminación, ¿es viable?}

Creo que es posible avanzar. Primero en experiencias piloto y mostrarle al mundo chileno que no debe tenerle miedo a eso. De hecho, la experiencia del proyecto 
del hospital Maquehue muestra que es posible administrar, conseguir y crear una experiencia de autonomía. Ello muestra que somos, como mapuche, capaces de gestionar, administrar. En la salud aquello también muestra que es posible con altos y bajos. La experiencia del borde costero lafkenche sigue mostrando que es posible. Hay varias experiencias. Yo diría que el Estado y la sociedad chilena deben darse cuenta de que la autonomía puede ser beneficiosa para todos. No sé, la autonomía es algo que me atrae, creo que puede ser un gran avance para Chile.

¿Cómo se convence al otro sector de la región, el que mayoritariamente no ve de manera positiva al mapuche en una región que, yo observo, tiene tan altos grados de racismo?

Es un tema cultural que se da no sólo con los mapuche. Por ejemplo, el tema del divorcio; demoró más de cien años en promulgarse una ley. El aborto en tres causales también es un tema cultural. El tema ambiental, la primera ley es de 1994. Todos son aspectos culturales.

Leí una entrevista a la concejala Genoveva Sepúlveda, miembro de la UDI, en el Austral de Temuco. Fuera de los argumentos para negar el derecho al aborto, no había leído otros argumentos demenciales como los que ella plantea públicamente. Además de señalar la amnistía a los militares condenados por violación a los derechos humanos -de hecho, protestó frente al Papa en Temuco-, sobre la lucha mapuche plantea sacar militares a la calle y otros argumentos que me hicieron imaginar que estaba viviendo el 11 de septiembre de 1973 a eso de las 13 horas. Senador: ¿cómo se crea un espacio pluricultural cuando existen personas con este tipo de ópticas y una población que ve con animadversión al movimiento mapuche?

En todos los sectores existen personas muy fanáticas que son muy difíciles de convencer. No se trata de obtener unanimidad; en los procesos sociales se trata de tener una gran mayoría. Nunca habrá unanimidad, es muy difícil ponerlos de acuerdo con todos, pero yo creo que en Chile tenemos muchos temas culturales pendientes. Es un trabajo lento, se ve en todos los ámbitos.

¿Entonces usted cree que el racismo se solucionaría solamente atacándolo desde una lógica cultural?

No, creo que es un tema de mentalidad. En otros países está más avanzado. Chile, sin embargo, es racista, muy clasista. Por eso creo que es cultural. Todos esos temas 
se han ido internalizando. Yo creo que hoy el movimiento mapuche ha tenido mucha fuerza y es respetado, hoy existe, salió a luz pública, se auto identifica: hay movimiento. Es un triunfo cultural que ha ido cambiando. Es un cambio positivo. Como senador, yo quiero trabajar en esa línea, de crear conciencia. Estar en los grandes temas nacionales, en los grandes debates, con una mirada mapuche. Por ejemplo, si Piñera dice "vamos modificar la Ley por Conductas Terroristas" agregando más ideas de seguridad, infiltración e inteligencia, yo no estaré de acuerdo. Porque la receta que ha fracasado es la de seguridad, el conflicto se ha tratado de orden público. El prisma debe ser político.

\section{¿Cuál es esa política?}

La política debe aceptar la realidad. Eso es lo primero. Reconocer que hubo un despojo. A partir de ello se puede comenzar por abrir lo otro. Pero seguir pensando que todo es de seguridad es un gran error. Ese camino está fracasado, es un remedio que no ha dado resultados.

Senador, hablemos de Bolivia. ¿Ve alguna lección que sacar de lo que ha construido el Estado Plurinacional de Bolivia para el caso mapuche, atendiendo que es una construcción "desde arriba”, una política "desde" el Estado?

No he seguido el proceso boliviano muy de cerca. Creo que la realidad de ellos es muy distinta, lo veo más parecido a Sudáfrica, de sincerar el Estado boliviano. Evo Morales me parece que sinceró el Estado boliviano, "no es un Estado blanco", dijo. Con los años, creo que ello va a pasar en Guatemala, Perú, Ecuador. Pero en Chile no es la situación, porque el mundo mapuche es minoría. La estructura social chilena es distinta a la boliviana. Por ende, los métodos del movimiento mapuche deben ser distintos.

Pero Bolivia ha construido un debate acorde a los acuerdos internacionales, adaptando las políticas internacionales a la realidad boliviana.

Ellos han creado ese sinceramiento. Tienen mayoría indígena. En Chile no tenemos mayoría para nada. Se debe crear una voluntad política de todos para avanzar en materias constitucionales, es un tema global a consecuencia de una Constitución engañosa en que la derecha tiene poder de veto. Entonces, estos mecanismos impiden poder efectuar reformas profundas. 


\section{$¿$ ¿Cree que se debe avanzar en un pacto que comprometa la plurinacionalidad?}

Chile debe, en ese espacio, hacer un trabajo de sinceramiento. Chile no existía, es el Estado el que construye la nación chilena. Y cuando el Estado se establece ya había pueblos anteriores a ello. Ese sinceramiento, Chile no lo ha hecho. Ahí están los gérmenes sociales de estos conflictos.

\section{¿Qué opina sobre que la oficialización del mapuzungun deba ser consultada de acuerdo a los lineamientos del Convenio 169 de la OIT?}

Mire, yo creo que es una de las cosas incomprensibles del siglo XXI. Bajo el sentido común uno habla como habla, yo no veo por qué se debe prohibir a una persona hablar en su idioma. Es sentido común. Eso no enriquece a la sociedad. Estos son remanentes de la Revolución Francesa, en que el Estado impone un idioma e identidad, pero hoy el 90\% de los Estados son plurinacionales. Eso es sentido común. Ahora, a mí me parece que no es aplicable, en este caso, el Convenio 169 de la OIT. Si uno hace una interpretación, el Convenio dice relación con proyectos de desarrollo que afecten a las comunidades o políticas que las afecten, pero hacer una consulta sobre la lengua es consultar sobre ti mismo. "Mire, ¿usted está de acuerdo en hablar como habla?" ¿Podrías consultar a Chile sobre hablar en castellano? Es una idea absurda. Es absurdo hacer una consulta para ello. En el fondo, lo que se busca es que los mapuche digan que no quieren hablar. El Convenio 169 de la OIT no puede consultar por un absurdo. Me parece absurdo.

La “Operación Huracán”, desde mi punto de vista, es el corolario de la "Operación Paciencia", aquel trabajo de inteligencia realizado bajo el gobierno de Ricardo Lagos. ¿Qué opinión tiene sobre este hecho?

Es la culminación de una política fracasada del Estado chileno durante estos últimos 20 años, que siempre vio los temas como de orden público y de seguridad. Si recuerdas, mientras fui intendente busqué cambiar y cambié la agenda en Chile porque señalé que este era un tema político y que por lo tanto la receta, aplicada a lo que sucedía en el sur de Chile, era una receta equivocada. Cuál era esa receta, te preguntarás. La receta de entregarle a Carabineros y al Ministerio Publico la solución de un tema político. La "Operación Huracán” es la culminación de una política equivocada, pero una política equivocada que se corrompió absolutamente.

Si analizamos estos últimos 20 años, desde mi punto de vista no se han visto resultados, entonces, el propio Estado se infligió una derrota, porque ya pasó de la investigación a la manipulación que le permite la ley para alterar las pruebas. Por 
estas cosas que pasan en la coyuntura de la historia se produjo este desacuerdo entre dos fuerzas del Estado, que lo ha llevado a su propia derrota. El hecho más grave es cursar pruebas y engaños, de parte del servicio de inteligencia de Carabineros, y también la ingenuidad del Gobierno de aceptar esas pruebas como tal. En ese ámbito, la institucionalidad del Estado chileno ha terminado haciendo el ridículo, independientemente del resultado final de esto. Porque todo esto podría terminar en que se revirtiera todo, dependiendo de los tribunales. Sin embargo, las confianzas están totalmente deterioradas, nadie va a creer los resultados de la investigación. Entonces, esto significó romper las confianzas, la credibilidad; el daño no es solamente a La Araucanía, es al país. ¿Qué pasaría si el país deja de creer en las instituciones? El país no funcionaría bien. El tema de fondo es que se han abierto las desconfianzas, la falta de credibilidad puede llevar al mundo mapuche a preguntarse, legítimamente: "bueno, este montaje, manipulaciones, ¿es actual? ¿También es pasado? ¿Cómo será en el futuro?” Por eso el Gobierno tiene una tremenda responsabilidad en tomar todas las medidas para la máxima transparencia y mostrar las cartas para el país, para que se sepa exactamente lo que ocurrió. Yo creo que hay un antes y un después en la credibilidad de instituciones como Carabineros. La tarea que tiene el Estado chileno es hoy altamente importante en torno a la credibilidad, tanto para este como para el gobierno que se aproxima.

\section{¿Cómo se revierte esta falta de confianza?}

Debe haber decisiones políticas drásticas. Partir por un cambio del alto mando y continuar con el establecimiento de las responsabilidades penales, administrativas y políticas. Una reestructuración y modernización de las policías, un cambio, digamos, en los servicios de inteligencia, en la forma en que entregan la información a las autoridades políticas. Honestamente, debe darse un cambio profundo en ese sentido. A mi parecer se debe comenzar por una reestructuración de la policía sí o sí. Pero no todo queda suscrito a ello, pensar que eso resolverá los problemas de La Araucanía es no observar la dimensión del problema.

Como lo he dicho, el problema de La Araucanía es un tema político y no se puede permitir que en un lugar del país la policía manipule pruebas. Eso abre caminos de desconfianzas no menores. Hoy son los mapuche, mañana podría ser la población migrante, la colonia árabe, no sé, la desconfianza no es buena para nadie. Ahí es donde veo la gravedad de lo que sucedió.

\section{Desde su óptica, ¿cómo debería haber respondido el Gobierno?}

Partir por cambiar al General Director de Carabineros y al conjunto del alto mando. Luego, una reestructuración con el fin de modernizar la institución y así poder 
entrar a la profundidad de ella para saber qué ocurrió de los mandos medios para abajo en los aspectos administrativos, en la formación interna de Carabineros. Debe darse una reestructuración de marca mayor, porque aquí lo que se quebrantó, en la historia de Chile, es la credibilidad y las confianzas.

Perdóneme una opinión: los gobiernos de la Concertación nos hicieron creer durante estos últimos años que Carabineros era una institución distinta a las policías de Latinoamérica: sin corrupción, justa y al servicio de la población. Sin embargo, el fraude al Fisco y la creación de pruebas, es decir, efectuar un delito para arrebatarle la libertad a la fuerza a dirigentes mapuche, me parece que es uno de los daños de imagen tal vez más profundos en una institución. Parece que no tenemos problemas y desafíos tan distintos de los del resto de las repúblicas latinoamericanas.

Yo creo que en la transición chilena hay un tema pendiente: la relación con la fuerza armada, sin "s", con la fuerza que en Chile tenemos armada, que son Carabineros e Investigaciones. Creo que existe un tema pendiente de la transición respecto a la fuerza armada a la que el Estado le ha entregado el monopolio legítimo de la fuerza. Hay niveles de autonomía que son inaceptables en un país democrático. Esto, en Europa, probablemente no sucedería. En Alemania no sucedería. Ello, porque la autonomía que se da es a partir de los manejos de recursos. Debemos recordar el Milico Gate. ¿Qué controles hay, por ejemplo, con la ley reservada del cobre? ¿Con la compra de armas? Hay un escenario a lo menos opaco en el nivel de autonomía de las Fuerzas Armadas en Chile, que está pendiente. Yo espero que a la hora de hablar de "seguridad" también hablemos de este tema.

Chile ha venido aplicando la Ley Antiterrorista a partir del año 2001, cuando se invocó por la quema de la casa de Juan Agustín Figueroa y un incendio a las plantaciones de forestal Mininco. Fue una decisión política aplicar esta ley de excepción para afrontar y enfrentar al movimiento mapuche. ¿Piensa que ese es el mecanismo para afrontar la demanda histórica de este pueblo?

Los políticos siempre recurren a esta ley cuando se da un hecho. Pero la Ley Antiterrorista ha sido aplicada muchas veces sin ningún resultado político. Ahora, que la legislación hay que modernizarla de acuerdo con los estándares internacionales, por supuesto. Todos los países tienen una legislación antiterrorista, pero debe ser hecha con los estándares que existen a nivel internacional en un Estado de derecho democrático, que respeta los derechos de la ciudadanía. Aquello está pendiente. 
Ariel Trangol está en huelga de hambre por el caso "Iglesias" y en estos momentos se desarrolla el juicio bajo la Ley por Conductas Terroristas y el juicio por el asesinato de los Lucshinger-Mackay está en una nueva etapa, luego de que la Corte fallara por repetir el caso. Hablemos de la huelga de hambre, un caso extremo de presión que el movimiento mapuche ha venido utilizando con fuerza como herramienta de protesta a partir del año 2006. ¿Cuál es su opinión sobre esto?

Mire, cuando fui autoridad, especialmente intendente, yo tenía una política que llamé "anticipación". Ella consistía en adelantarme, dialogar, iniciar conversaciones de inmediato, ya que el trabajo de una autoridad es conversar, adelantarse a los hechos que pueden causar conflictos políticos. La huelga de hambre es un conflicto político y por lo tanto la autoridad debería dialogar, analizar los términos y escuchar por qué esa persona ha puesto en riesgo su vida. Eso se logra dialogando, conversando. Luego la autoridad podrá ponderar, pero antes que todo debemos dialogar. Cuando fui intendente practiqué esto y tuve muy buenos resultados de mi gestión, ya que logramos adelantarnos a los problemas, buscar soluciones y continuar luego con normalidad con el fin de revertir las desconfianzas. Si Ariel Trangol no ha tenido diálogo con el Gobierno me parece preocupante, o si el Gobierno no ha tenido diálogos con Trangol me parece igual de preocupante.

Su visión sobre la prisión política mapuche: ¿cree que afecta las confianzas?

¿Es que sabes lo que pasa? Si la Corte Suprema dejó libre a todas las personas de la "Operación Huracán", lo que uno siempre quisiera es creer que los tribunales dirán "a" o "b". En el último tiempo los tribunales han tenido distintos fallos y como abogado, yo siempre he tenido una mirada sobre el Poder Judicial bastante positiva, porque creo que si la Corte Suprema es capaz de dar una resolución como lo hizo en la "Operación Huracán" es positivo, no porque los deje libres, que también es positivo, sino porque un tribunal es capaz de sustraerse de las presiones para dictar un fallo unánime. Yo no estoy pidiendo para los mapuche un trato especial, estoy pidiendo un trato que se le da al conjunto de la población, ello se llama presunción de inocencia. Ella debe ser para todos. Eso es positivo. Un fallo a favor de "Huracán" reestablece las confianzas en los tribunales. A mi parecer, poder llegar a la Corte Suprema debería ser visto por los mapuche como un acto positivo, ya que verlo como la crónica de algo adverso implicaría desconfianzas brutales, pero en este caso se reafirman las posibilidades para Chile de tener un Poder Judicial que se sobreponga a todas las presiones. Eso es lo que necesitamos. 


\section{Usted será senador ocho años, cuatro de ellos con Sebastián Piñera como Presidente. ¿Cómo piensa que será este segundo gobierno?}

En materia indígena será difícil. Creo que este Gobierno tendrá dos caras: una económica y otra política. En esta segunda será un escenario difícil, pues este Gobierno tiene una visión tal vez de volver atrás en materias como Ley Indígena, Conadi, compra de tierras y el Convenio 169 de la OIT. Se aleja aún más el reconocimiento constitucional. Si a ello agregamos el tema de la seguridad, será un escenario complejo y difícil.

\section{¿Más que bajo los gobiernos de la Concertación y la Nueva Mayoría?}

Sí, porque una cara de la derecha es lo que te señalaba. Pero en la otra cara pienso que la derecha pondrá muchos recursos a La Araucanía y por eso se explica la nominación en el Ministerio de Desarrollo Social de un alto miembro de los empresarios. Para mí esto apunta a que se le dará prioridad a una perspectiva económica en la región. A mi juicio, si queremos una solución integral, insoslayablemente debemos tomar ambos aspectos, no basta sólo con el desarrollo, deben resolverse los temas políticos, si no es esconder el problema bajo la alfombra sin analizar el fondo: los derechos de las primeras naciones. No verlo es jugar contra la historia. Esta derecha conservadora que ve un país uniforme, racialmente unido, no acepta la diversidad, piensa que somos una sola nación y no ve en la diversidad un valor, lo ve como una disminución del rol del Estado. Ellos ven una nación con un solo Estado. Y eso es no querer encarar el problema, lo que traerá nuevos problemas aquí y en el futuro. Para mí no basta con más recursos.

\section{Para ir cerrando, senador, ¿cómo evalúa el Gobierno de Michelle Bachelet del que usted también fue parte?}

Bueno, yo soy parte de esa coalición. Me parece que la Presidenta comenzó un proceso de reformas que a mi juicio iban en la dirección correcta para reestructurar el modelo neoliberal que tenemos en Chile. A mi juicio, el principal déficit de este gobierno fue de gestión política, hubo muchos problemas políticos en la implementación de las reformas y muchos problemas de déficit político en la conducción. Los problemas del Gobierno de la Presidenta Bachelet fueron de gestión política.

En materia indígena se hizo más de lo mismo, se centró todo en las políticas públicas, se siguió el mismo camino ya hecho en los periodos anteriores. ¿Qué quiere decir esto? Analizar la demanda indígena como algo judicial, evitando un cambio 
de eje, lamentablemente; es decir, analizarlo desde una perspectiva política. Así que yo creo que se siguió con una política de hace 20 años y no se entró en los temas de fondo. En síntesis, los problemas de este gobierno fueron de déficit político y de continuar haciendo lo mismo en materia mapuche y derechos indígenas.

\section{A su juicio, entonces: ¿cuál ha sido el gobierno que ha buscado abordar de mejor forma las demandas indígenas en materia de políticas públicas?}

El gobierno de Patricio Aylwin sentó las bases de lo que han sido las políticas de los distintos gobiernos hasta la fecha. Fue bajo su gobierno que se dictó la Ley Indígena de 1993, ella es la base de todas las políticas públicas que se han hecho en los gobiernos posteriores. Ella es obra del Presidente Aylwin. Sobre eso, los gobiernos posteriores siguieron haciendo más de lo mismo. Tal vez la excepción sea la ratificación del Convenio 169 de la OIT, realizado bajo el primer gobierno de la Presidenta Michelle Bachelet, pero yo creo que ese Convenio tuvo un error de base: se aprobó sin que la derecha y los sectores empresariales tuvieran la voluntad de hacerlo. Yo creo que ese mundo de la derecha y empresarios ha cometido un error, porque sin voluntad política el Convenio 169 tendrá puras dificultades. Y así lo hemos visto. En otros países, con voluntad política, las relaciones son mucho mejores. Ese es el problema que tiene el Convenio 169 de la OIT.

\section{¿Qué nos falta a los pueblos originarios para desarrollar una nueva política?}

No hay una nueva política sin la voluntad y el convencimiento de la clase política y la empresarial de encarar el problema desde otras aristas. Yo no veo ese conocimiento ni el convencimiento, menos la voluntad política de un cambio de paradigma en las clases dirigentes chilenas. Hablo de todos. Nadie tiene esa voluntad política y sin eso vamos a seguir en más de lo mismo. La tarea en mis ocho años es contribuir a cambiar esa voluntad política y espero lograrlo. 